\title{
BMJ Open Health-related preferences of older patients with multimorbidity: the protocol for an evidence map
}

\author{
Ana Isabel González-González, ${ }^{1,2}$ Christine Schmucker, ${ }^{3}$ Jeanet Blom, ${ }^{4}$ \\ Marjan van den Akker, ${ }^{1}$ Truc Sophia Nguyen, ${ }^{1}$ Julia Nothacker, ${ }^{3}$ Joerg J Meerpohl, ${ }^{3}$ \\ Kristian Röttger, ${ }^{5}$ Odette Wegwarth, ${ }^{\circ}$ Tammy Hoffmann, ${ }^{7}$ Sharon E Straus, ${ }^{8}$ \\ Ferdinand M Gerlach, ${ }^{1}$ Christiane Muth ${ }^{1}$
}

To cite: González-González Al, Schmucker C, Blom J, et al. Health-related preferences of older patients with multimorbidity: the protocol for an evidence map. BMJ Open 2019;9:e029724. doi:10.1136/ bmjopen-2019-029724

- Prepublication history and additional material for this paper are available online. To view these files, please visit the journal online (http://dx.doi. org/10.1136/bmjopen-2019029724).

Received 11 February 2019 Revised 21 June 2019 Accepted 06 August 2019

Check for updates

(C) Author(s) (or their employer(s)) 2019. Re-use permitted under CC BY-NC. No commercial re-use. See rights and permissions. Published by BMJ.

For numbered affiliations see end of article.

\section{Correspondence to}

Dr Ana Isabel González-

González;

gonzalezgonzalez@

allgemeinmedizin.uni-frankfurt. de

\section{ABSTRACT}

Introduction Interaction of conditions and treatments, complicated care needs and substantial treatment burden make patient-physician encounters involving multimorbid older patients highly complex. To optimally integrate patients' preferences, define and prioritise realistic treatment goals and individualise care, a patient-centred approach is recommended. However, the preferences of older patients, who are especially vulnerable and frequently multimorbid, have not been systematically investigated with regard to their health status. The purpose of this evidence map is to explore current research addressing health-related preferences of older patients with multimorbidity, and to identify the knowledge clusters and research gaps.

Methods and analysis To identify relevant research, we will conduct searches in the electronic databases MEDLINE, EMBASE, PsycINF0, PSYNDEX, CINAHL, Social Science Citation Index, Social Science Citation Index Expanded and the Cochrane library from their inception. We will check reference lists of relevant articles and carry out cited reference research (forward citation tracking). Two independent reviewers will screen titles and abstracts, check full texts for eligibility and extract the data. Any disagreement will be resolved and consensus reached with the help of a third reviewer. We will include both qualitative and quantitative studies, and address preferences from the patients' perspectives in a multimorbid population of 60 years or older. There will be no restrictions on the publication language. Data extraction tables will present study and patient characteristics, aim of study, methods used to identify preferences and outcomes (ie, type of preferences). We will summarise the data using tables and figures (ie, bubble plot) to present the research landscape and to describe clusters and gaps.

Ethics and dissemination Due to the nature of the proposed evidence map, ethics approval will not be required. Results from our research will be disseminated by means of specifically prepared materials for patients, at relevant (inter)national conferences and via publication in peer-reviewed journals.

\section{INTRODUCTION}

Multimorbidity is defined as the co-occurrence of two or more acute or chronic diseases

\section{Strengths and limitations of this study}

- Strengths of the study include, first, the considerable expertise, methodological experience and skills that result from having a multinational and multidisciplinary study team that also includes a patient's representative.

- Second, the search will be broad based, use a sensitive rather than a specific strategy and cover a wide range of databases, terms and search strategies (eg, forward citation tracking).

- Third, selection criteria will also be broad (ie, both qualitative and quantitative studies will be considered) and no restrictions will be placed on setting or language of publication.

- The main study limitation is poor indexing of articles and the lack of, or non-standardised definition, of 'patient preferences' (eg, expressed as satisfaction, experience or perspectives).

- The planned evidence map is expected to help researchers identify clusters and gaps in evidence on preferences of older patients with multimorbidity.

and medical conditions in one person. ${ }^{1}$ The prevalence of multimorbidity increases significantly with age, rising from about $50 \%$ at the age of 60 years to more than $80 \%$ at the age of 80 , although the estimates vary widely depending on the employed definition of multimorbidity. ${ }^{2-7}$ Interaction of conditions and treatments, complicated care needs and substantial treatment burden make patientphysician encounters involving multimorbid older patients highly complex, and the clinical management of these patients extremely challenging. ${ }^{8-10}$

Although interventions to improve relevant outcomes in older patients with multimorbidity still lack high-quality evidence, ${ }^{11} 12$ existing principles, ${ }^{13}$ clinical practice guidelines, ${ }^{14}$ recommendations for research ${ }^{9}$ and care models, ${ }^{15}$ all recommend a patient-centred approach that takes patient preferences 
into consideration. Multimorbidity can be associated with overwhelming management burden, which makes it necessary for physicians and patients to prioritise treatment plans by considering both the reduction of symptoms and the patients' quality of life. ${ }^{16}{ }^{17}$ As every treatment option consists of a specific combination of benefits, harms and burden, it is important that physicians understand the need to take older patients' preferences and priorities into account in the decision-making process. Tailoring treatments to each individual older patient's needs and preferences is likely to improve adherence to self-management interventions and medication. ${ }^{18}$

The GRADE working group defines preferences as choices that patients make when 'considering the potential benefits, harms, costs, limitations and inconveniences of the management options in relation to one another' ${ }^{19}$ Overall, preferences include patients' beliefs, expectations, desires, perspectives and goals. ${ }^{19}$ Certain preferences, such as the avoidance of pain, are stable and well articulated by patients. However, most preferences relating to the medical decision-making process have to be broken down into their individual components, as the patient is often not familiar with them. For example, the potential benefits and harms of a new drug treatment have to be taken into consideration and weighed against each other and across diseases, especially in older patients with multimorbidity. The elucidation and construction of preferences is a complex process that several disciplines have investigated from different perspectives. ${ }^{20-23}$

Healthcare decision-making in multimorbidity requires that health problems are prioritised in terms of desired versus undesired outcomes-a situation that patients often have no experience with. ${ }^{24}$ Clinical decision elements may be unfamiliar to them, and the available choices may present a conflict in that one goal can only be achieved by forgoing another. ${ }^{21}$ Moreover, multimorbidity is often characterised by a state of shifting priorities in self-management that can change from day to day. ${ }^{25}$ Hence, most healthcare-related preferences must be constructed during a process of elicitation that is part of the decision-making process. ${ }^{21}$

Although several tools have been developed to assess multimorbid patients' preferences (eg, for different treatment options or outcomes) in terms of the prioritisation of their health-related goals, ${ }^{26}$ no structured attempt has yet been made to summarise the current state of research on healthcare-related preferences in this patient population. However, the broad nature of this topic requires that existing evidence is mapped out, that is, a systematic search of existing knowledge in the field should be conducted to identify gaps and/or future research needs. $^{27}$

In this article, we report the protocol of an evidence map to: (1) systematically identify and describe key characteristics of research on health-related preferences of older patients with multimorbidity, (2) display the existing research landscape in visual formats, (3) identify evidence clusters to guide subsequent knowledge synthesis (systematic reviews and meta-analysis) and (4) identify evidence gaps to inform patients, clinicians, researchers, policy-makers and funding agencies, and to help identify future research priorities. This work will provide us with a thorough overview of research on the health-related preferences of older patients with multimorbidity.

\section{METHODS AND ANALYSIS}

Evidence mapping is an innovative method of synthesising evidence that is particularly useful when the research question is too broad to permit a 'traditional' systematic review to be performed. Evidence maps have recently been recommended by the Agency for Healthcare Research and Quality's Evidence-based Practice Center $\operatorname{program}^{28}$ as a first step towards systematically mapping existing research (clusters and gaps in evidence) that can help answer broad-based questions. They usually use visual formats (eg, bubble plots) to analyse and present results. ${ }^{29}$

The aim of the evidence maps is to "collate, describe and catalogue' knowledge of a broad subject area. ${ }^{27} 30$ Evidence maps are particularly effective when research questions are wide-ranging because they explore rather than summarise evidence. Consequently, the evidence maps do not include meta-analysis or compare the strength of evidence between studies but chart concepts, themes and the amount and type of evidence available.

The present protocol will follow, where applicable, the 'Preferred Reporting Items for Systematic Reviews and Meta-Analyses Extension for Scoping Reviews' checklist ${ }^{31}$ (see online additional file 1).

Following the framework originally establish by Arksey and O'Malley, ${ }^{32}$ refined by Levac $e t a l^{33}$ and further developed by the Joanna Briggs Institute, ${ }^{34}$ six steps will be used to create the evidence map: (1) identifying a broad clinical question, (2) identifying relevant studies, (3) study selection, (4) charting the data, (5) reporting the results and (6) consultation.

\section{Step 1: identifying a broad clinical question}

A pilot test of an evidence map for our research question (published elsewhere) was performed as part of a collaboration between the Institute of General Practice at Johann Wolfgang Goethe University (Frankfurt) and the Institute for Evidence in Medicine (for Cochrane Germany Foundation), Freiburg. It showed the feasibility of the mapping approach and areas for improvement, thus helping to refine the research question and the methods to be used.

We established a multidisciplinary research team of 11 experts-some of whom had more than one area of expertise-from five countries (Australia, Canada, Germany, Spain and The Netherlands). In addition to a patient representative (one) the professionals represented primary care (two), internal medicine (one), geriatrics (one), cognitive psychology (one), public health and health services research (two), methodology (three), 
shared decision-making (one), epidemiology (one) and knowledge translation (one).

At the project kick-off meeting in April 2018, all members of the multidisciplinary research team contributed to the definition of the scope of the evidence map. Based on the results of previous exploratory research, we defined the following question to be addressed by our evidence map: What specific health-related preferences of older patients with multimorbidity are described in the available literature?

\section{Step 2: identifying relevant studies}

In order to identify relevant published studies, we will conduct a literature search in the following electronic databases: MEDLINE (1946 to 2018) via Wolters Kluwer's search interface Ovid (indexed and non-indexed databases), CINAHL (1981 to 2018), PsycINFO (1800s to 2018) and PSYNDEX via EBSCOhost, Science Citation Index Expanded (1945 to 2018) and Social Science Citation index (1956 to 2018) via Clarivate Analytics' Web of Science and EMBASE (1988 to 2018) via Ovid, and Cochrane Database (CENTRAL, TRIALS). We will check the reference lists of included articles (backward citation tracking) and carry out forward citation tracking using the Web of Science Core Collection. Additionally, we will search for related articles in PubMed. Authors of conference proceedings that have not published a full set of results will be contacted. Secondary research (ie, systematic reviews, synthesis of qualitative studies and scoping reviews) studies on related topics will be reviewed and references will be checked for possible inclusion in the evidence map. We will also search for ongoing trials in ClinicalTrials.gov and WHO register.

We followed the recommendations of PRESS Peer Review of Electronic Search Strategies and developed the final search strategy in collaboration with an expert medical sciences librarian. ${ }^{35}$

The full electronic search strategy for the MEDLINE database is provided in online additional file 2.

Based on the results of pilot testing, we agreed with all collaborative partners on the following eligibility criteria for the evidence map during the kick-off meeting in April 2018 (see table 1):

\section{Participants/population}

Older patients (mean and/or median age $\geq 60$ years) with multimorbidity (two or more simultaneous acute or chronic conditions ${ }^{1}$ ) of any type will be considered.

\section{Outcomes}

Our phenomena of interest (outcomes) will be: (1) preferences related to the organisation of healthcare, (2) preferences for specific information, communication or involvement in a shared decision-making process, (3) preferences relating to desired, undesired and competing outcomes (in terms of safety and effectiveness), (4) prioritisation of health problems or conditions, (5) screening or diagnostic procedure preferences and (6) treatment preferences. The classification of the outcomes will be discussed and consecutively adapted, depending on the literature findings. This classification will further allow content analysis and the establishment of research clusters and gaps.

\section{Study setting}

We will not apply any restriction to the geographical location of the study or the language of publication, and we will include studies conducted in any setting, that is, any healthcare context in any country (including low-income and middle-income countries).

\section{Study design}

We will include qualitative and quantitative studies that address the phenomena of interest defined above from the patients' perspectives.

We will exclude case reports, narrative reviews and editorials. We will leave out studies investigating preferences for or against interventions of limited availability or whose legal status is unclear (eg, euthanasia, which is neither legal nor available in most Western countries). Studies addressing only the preferences of caregivers, family or medical and/or other professionals will not be considered.

\section{Step 3: study selection}

Bibliographic details of all identified references will first be uploaded to EndNote and then converted into Covidence, which will automatically detect duplicate

Table 1 Inclusion and exclusion criteria

\begin{tabular}{ll}
\hline Inclusion criteria & Exclusion criteria \\
\hline Qualitative and quantitative studies addressing health-related & Case reports. \\
preferences (priorities, goal oriented, goal attainment, shared & Narrative reviews. \\
decision-making, patient centred, patient oriented, 'satisfaction') & Editorials. \\
from the patient's perspective. & Studies investigating preferences for or against interventions \\
Age: average age of 60 or older, geriatric patients, elderly & contexts (eg, euthanasia). \\
patients. & Studies addressing only preferences of caregivers and \\
Polypharmacy: with or without polypharmacy. & \\
Multimorbidity: comorbidity, multiple chronic conditions. & \\
No restrictions: we will not apply any restrictions to the & \\
geographical location, healthcare context, country and & \\
publication language of the study. &
\end{tabular}


Table 2 Data extraction framework

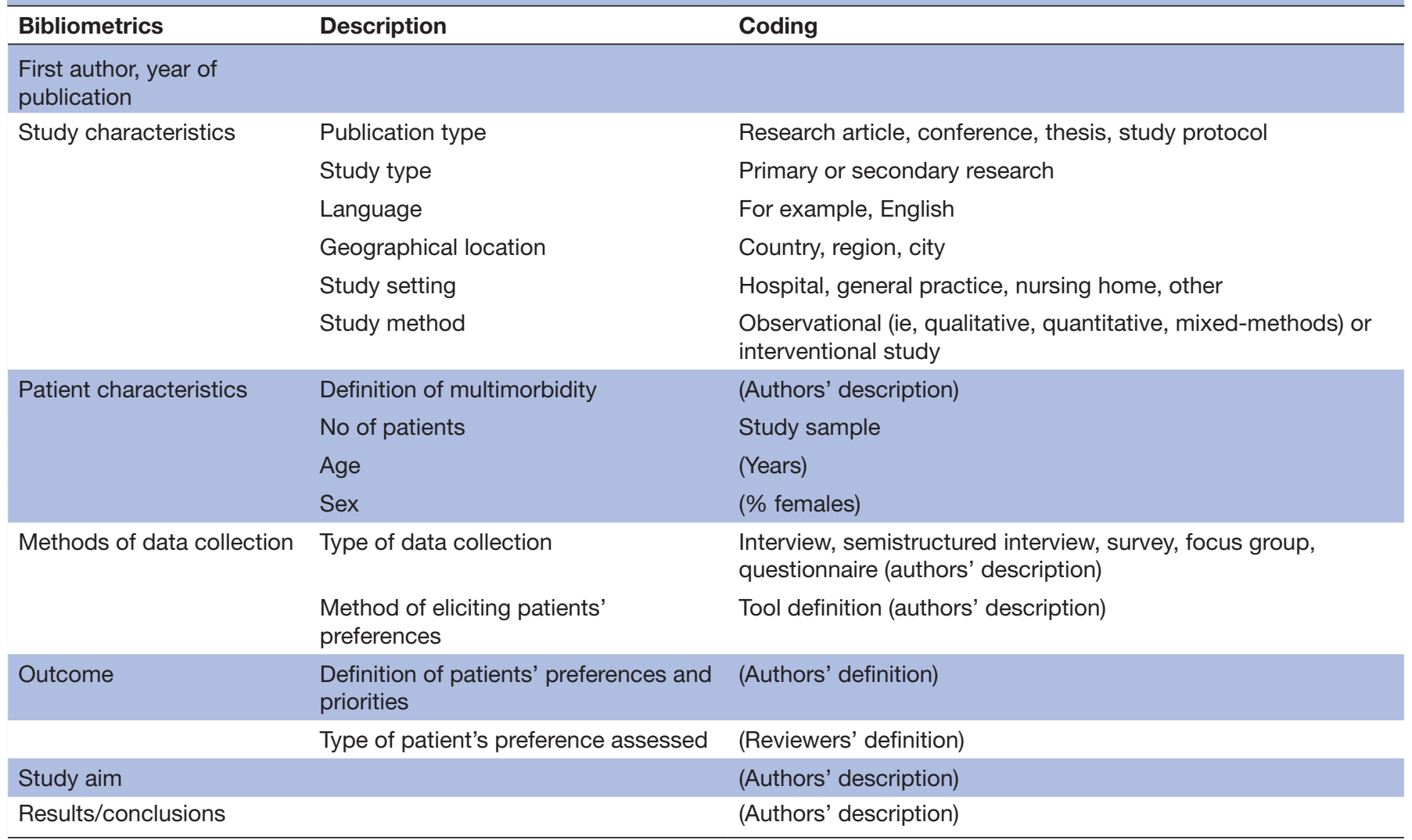

documents. Two reviewers (AIG, JN) will independently screen titles and abstracts and will independently check full texts of the included articles for eligibility. Any disagreement will be resolved and consensus reached with the help of a third reviewer (CS). Before screening, a stepwise calibration exercise will be performed on a sample of 50 studies, with the aim of achieving $80 \%$ agreement between the two reviewers. In case $80 \%$ agreement is not reached, our inclusion and exclusion criteria will be refined to reach this cut-off (eg, defined more stringently). Refined criteria will be calibrated on a new sample of 50 studies and repeated until this threshold is reached. We will report any changes to the inclusion and exclusion criteria that result from the calibration exercise as deviations from the published protocol.

\section{Step 4: charting the data}

Data extraction tables will be created using Excel and will include, when available: study characteristics such as research type (study design/methodology) and setting (healthcare context, country of origin and study period); patient characteristics (sample size, age, sex and definition of multimorbidity); aim of study; characteristics of the preferences, such as methods used to elucidate patients' preferences, framing and definition of preferences (eg, treatment preferences, diagnostic preferences, desired, undesired and competing outcome preferences-as guided by the above description of the phenomena of interest) and results (see table 2 ).

Following a calibration exercise on five full texts, two reviewers (AIG, JN or CS) will independently extract the data. To check the adequacy of the extracted information, the data extraction file will be shared with other authors (CM, JB, MvdA, TH and SES), and changes performed where necessary.

\section{Step 5: reporting the results}

We will summarise the data using tables and figures (ie, bubble plot) to present the evidence landscape and to elucidate clusters and gaps. For each year, we will identify the number of primary and secondary research studies, as well as conferences and doctoral theses, which describe patients' preferences. We will describe the identified studies in terms of characteristics such as location, setting and study design (ie, observational-qualitative, quantitative or mixed-methods-or interventional studies), subpopulation according to age or multimorbidity pattern/severity if possible, and study objectives aggregated according to research topic (ie, type of preference) (table 2).

Clustering of research topics will be performed by applying content analysis ${ }^{36} 37$ to summarise the types of preference described in the study. Based on coding by two independent reviewers (AIG, JN or CS), overarching 
themes will be identified and aggregated. For this purpose, the results will be entered into the data extraction file, which will then be reviewed by the other researchers (CM, JB, MvdA, TH and SES). Categories for the analysis of the obtained data will be modified accordingly, along with the development of the evidence map, and agreed on after consultation with the research team.

\section{Step 6: consultation}

The development of the evidence map will follow an iterative process and all members of the research team will be consulted during all steps of the project, including the identification of relevant literature, study selection and data extraction. In November 2018, we held a workshop to present the results of the preliminary search strategy and exploratory investigation, and to obtain feedback before conducting further searches and other activities. We discussed interim results, refined the methodology and agreed on the best formats for reporting our findings. Cluster definitions of the identified research topics were discussed and agreed on by all authors. All necessary changes were established before continuing with the development of the evidence map.

The present study started on 1 February 2018 and is scheduled to end on 31 October 2019.

\section{Patient and public involvement}

A patient representative (KR) from the Federal Joint Committee 'Gemeinsamer Bundesausschuss (G-BA)' will actively participate in all six steps required to create the evidence map. As a result of his work on the G-BA board of patients' representatives, KR has considerable expertise in evidence-based medicine in a healthcare context, and an understanding of the pivotal role of patients' preferences in the provision of effective healthcare. The G-BA constitutes the highest decision-making body for the joint self-administration of stakeholders in the German health service, and the statutory health insurance service catalogue for over 70 million insured individuals is based on its guidelines.

\section{ETHICS AND DISSEMINATION}

We prepare presentations to disseminate the study findings to healthcare providers and patients, and at relevant national and international conferences, and we aim to publish the results of the study in peer-reviewed journals. We will provide recommendations for primary research that are based on the identified knowledge gaps, and recommendations for secondary research that are based on knowledge clusters.

\footnotetext{
Author affiliations

${ }^{1}$ Institute of General Practice, Johann Wolfgang Goethe University, Frankfurt, Germany

${ }^{2}$ Red de Investigación en Servicios de Salud en Enfermedades Crónicas (REDISSEC), Madrid, Spain

${ }^{3}$ Institute for Evidence in Medicine (for Cochrane Germany Foundation), Medical Center-University of Freiburg, Faculty of Medicine, University of Freiburg, Freiburg, Germany
}

${ }^{4}$ Department of Public Health and Primary Care, Leiden University Medical Center, Leiden, The Netherlands

${ }^{5}$ Patient Representative, Federal Joint Committee "Gemeinsamer

Bundesausschuss", Berlin, Germany

${ }^{6}$ Center for Adaptive Rationality, Max Planck Institute for Human Development, Berlin, Germany

${ }^{7}$ Institute for Evidence-Based Healthcare, Faculty of Health Sciences and Medicine, Bond University, Gold Coast, Queensland, Australia

${ }^{8}$ Department of Medicine, University of Toronto, Toronto, Ontario, Canada

Acknowledgements The authors thank the expert medical science librarian, Edith Motschall (Institute of Medical Biometry and Statistics, Faculty of Medicine, University of Freiburg, GERMANY), for her contribution developing and carrying out the search strategy. We would like to thank as well Katharina Kunzweiler for supporting us in the literature screening process.

Contributors AIG wrote the initial draft of the protocol. CM is the guarantor of the review. CS and JJM provided methodological guidance and revisions of the manuscript. CS and JN assisted in the identification of databases and reviewed the search strategy. JB, MvdA, TSN, JN, OW, KR, TH, FMG and SES are cosupervisors of this project, provided advice at all stages of the development of the protocol, and contributed to the revision of the manuscript. All authors read and approved the final manuscript.

Funding This work was supported by the German Federal Ministry of Education and Research, grant number 01GL1729.

Disclaimer The funder had no role in developing the protocol for this review.

Competing interests None declared.

Patient consent for publication Not required.

Ethics approval Due to the nature of the proposed evidence map, ethics approval will not be required.

Provenance and peer review Not commissioned; externally peer reviewed.

Open access This is an open access article distributed in accordance with the Creative Commons Attribution Non Commercial (CC BY-NC 4.0) license, which permits others to distribute, remix, adapt, build upon this work non-commercially, and license their derivative works on different terms, provided the original work is properly cited, appropriate credit is given, any changes made indicated, and the use is non-commercial. See: http://creativecommons.org/licenses/by-nc/4.0/.

\section{REFERENCES}

1. van den Akker M, Buntinx F, Knottnerus JA. Comorbidity or multimorbidity. Eur J Gen Pract 1996;2:65-70.

2. van den Akker M, Buntinx F, Metsemakers JFM, et al. Multimorbidity in general practice: prevalence, incidence, and determinants of co-occurring chronic and recurrent diseases. J Clin Epidemiol 1998;51:367-75.

3. Violan C, Foguet-Boreu Q, Flores-Mateo G, et al. Prevalence, determinants and patterns of multimorbidity in primary care: a systematic review of observational studies. PLoS One 2014;9:e102149-11.

4. Wolff JL, Starfield B, Anderson G. Prevalence, expenditures, and complications of multiple chronic conditions in the elderly. Arch Intern Med 2002;162:2269-76.

5. Hoffman C. Persons with chronic conditions. JAMA 1996;276.

6. Fortin M, Bravo G, Hudon C, et al. Prevalence of multimorbidity among adults seen in family practice. Ann Fam Med 2005;3:223-8.

7. Marengoni A, Angleman S, Melis R, et al. Aging with multimorbidity: a systematic review of the literature. Ageing Res Rev 2011;10:430-9.

8. Burgers JS, Voerman GE, Grol R, et al. Quality and coordination of care for patients with multiple conditions: results from an international survey of patient experience. Eval Health Prof 2010;33:343-64.

9. Bayliss EA, Bonds DE, Boyd CM, et al. Understanding the context of health for persons with multiple chronic conditions: moving from what is the matter to what matters. Ann Fam Med 2014;12:260-9.

10. Bayliss EA, Edwards AE, Steiner JF, et al. Processes of care desired by elderly patients with multimorbidities. Fam Pract 2008;25:287-93.

11. Smith SM, Wallace E, O'Dowd T, et al. Interventions for improving outcomes in patients with multimorbidity in primary care and community settings. Cochrane Database Syst Rev 2016;9.

12. Smith SM, Soubhi H, Fortin M, et al. Managing patients with multimorbidity: systematic review of interventions in primary care and community settings. BMJ 2012;345:e5205. 
13. Muth $\mathrm{C}$, van den Akker M, Blom JW, et al. The Ariadne principles: how to handle multimorbidity in primary care consultations. BMC Med 2014;12:223.

14. Muth C, Blom JW, Smith SM, et al. Evidence supporting the best clinical management of patients with multimorbidity and polypharmacy: a systematic guideline review and expert consensus. J Intern Med 2019;283:272-88.

15. Palmer K, Marengoni A, Jureviviene E, et al. Multimorbidity care model : Recommendations from the consensus meeting of the Joint Action on Chronic Diseases (CHRODIS), 2016.

16. Kastner M, Hayden L, Wong G, et al. Underlying mechanisms of complex interventions addressing the care of older adults with multimorbidity: a realist review. BMJ Open 2019;9:e025009.

17. Kastner M, Cardoso R, Lai Y, et al. Effectiveness of interventions for managing multiple high-burden chronic diseases in older adults: a systematic review and meta-analysis. Can Med Assoc J 2018;190:E1004-12.

18. Pagès-Puigdemont $\mathrm{N}$, Mangues MA, Masip M, et al. Patients' Perspective of Medication Adherence in Chronic Conditions: A Qualitative Study. Adv Ther 2016;33:1740-54.

19. Andrews JC, Schünemann HJ, Oxman AD, et al. Grade guidelines: 15. going from evidence to recommendation-determinants of a recommendation's direction and strength. J Clin Epidemiol 2013;66:726-35

20. Ormel J, Lindenberg S, Steverink N, et al. Subjective well-being and social production functions. Soc Indic Res 1999;46:61-90.

21. Lichtenstein S, Slovic P. The construction of preference. Cambridge; New York: Cambridge University Press, 2006.

22. Fishbein M, Ajzen I. Belief, attitude, intention and behaviot: an introduction to theory and research, 1975: $578 \mathrm{p}$

23. Franz S. Grundlagen des ökonomischen Ansatzes: Das Erklärungskonzept des Homo Oeconomicus, 2004.

24. Hansen $\mathrm{H}$, Pohontsch $\mathrm{N}$, van den Bussche $\mathrm{H}$, et al. Reasons for disagreement regarding illnesses between older patients with multimorbidity and their GPs - a qualitative study. BMC Fam Pract 2015;16:68.
25. Morris RL, Sanders C, Kennedy AP, et al. Shifting priorities in multimorbidity: a longitudinal qualitative study of patient's prioritization of multiple conditions. Chronic IIIn 2011;7:147-61.

26. Mangin D, Stephen G, Bismah V, et al. Making patient values visible in healthcare: a systematic review of tools to assess patient treatment priorities and preferences in the context of multimorbidity. BMJ Open 2016;6:e010903.

27. Miake-Lye IM, Hempel S, Shanman R, et al. What is an evidence MAP? A systematic review of published evidence maps and their definitions, methods, and products. Syst Rev 2016;5:28.

28. Chang S, Reviews S. Scoping reviews and systematic reviews: is it an either/or question? Ann Intern Med 2018;169:502.

29. Schmucker C, Motschall E, Antes G, et al. Methoden des Evidence Mappings. Bundesgesundheitsblatt - Gesundheitsforsch. Gesundheitsschutz 2013;56:1390-7.

30. James KL, Randall NP, Haddaway NR. A methodology for systematic mapping in environmental sciences. Environ Evid 2016;5.

31. Tricco AC, Lillie E, Zarin W, et al. PRISMA extension for scoping reviews (PRISMA-ScR): checklist and explanation. Ann Intern Med 2018;169:467.

32. Arksey H, O'Malley L. Scoping studies: towards a methodological framework. Int J Soc Res Methodol 2005;8:19-32.

33. Levac D, Colquhoun H, O'Brien KK. Scoping studies: advancing the methodology. Implementation Sci 2010;5

34. The Joanna Briggs Institute. The Joanna Briggs Institute Reviewers' manual 2015 - methodology for JBI scoping reviews. Adelaide, South Australia, 2015

35. McGowan J, Sampson M, Salzwedel DM, et al. CADTH methods and guidelines, 2016

36. Vaismoradi $\mathrm{M}$, Turunen $\mathrm{H}$, Bondas $\mathrm{T}$. Content analysis and thematic analysis: implications for conducting a qualitative descriptive study. Nurs Health Sci 2013;15:398-405.

37. Krippendorff K. Content analysis: an introduction to its methodology. 2nd ed. Thousand Oaks, CA: Sage, 2004: 413 p. 